\section{COMPARATIVE VALUE OF IPECAC AND ITS ALKALOIDS IN TREATMENT OF INTESTINAL ENDAMEBIASIS *}

\author{
SIDNEY K. SIMON, M.D.
}

Assistant Professor of Clinical Medicine, Tulane University of Louisiana NEW ORLEANS

There is universal concurrence of opinion at the present time in regard to the highly specific effect of ipecac, as well as of its constituent alkaloids, on the vegetative forms of the pathogenic endameba. Though the ipecacuanha plant, and especially its root, has been employed since remote times as an empiric remedy in the treatment of many varieties of intestinal disorders, it is only within comparatively recent years that recognition of its sole and distinctive value in endamebic disease has been definitely reached.

An interesting and diversified history surrounds the earlier use of ipecac as a therapeutic agent, traceable back as far even as the beginning of the Middle Ages. It was first introduced into Europe in 1648 in the guise of a secret remedy for dysentery, having gained considerable repute originally among the natives of Brazil for its usefulness in this class of affections. The story is related that the French government, impressed by the striking results obtained in the case of the Dauphin, son of Louis XIV, then reigning, was induced to purchase the secrets of the wonderful remedy outright, for universal dissemination. In the course of the years that have since followed, reaching to the present day, ipecac, in some one of its many forms, has weathered a shifting reputation for dependability in dysenteric disturbances, being lauded and denounced in turn by various authorities in almost as many climes.

During the period immediately preceding the close of the last century, the drug had fallen into almost complete disuse, doubtleșs because of the disagreeable effects which so often attended its administration. In the eyes of the more careful English observers in the tropics, however, ipecac had always maintained the foremost place as an indispensable measure for combating the dysenteries of warm countries, especially in India, and it is, in fact, due largely to the earnest pleas of this school, headed by the venerable Sir Patrick Manson, that credit should be accorded for rescuing the drug within recent years from all but entire oblivion.

Some ten years ago, being impressed by the weight of this authoritative experience and especially after I had previously convinced myself of the noneffectiveness of the various measures then in vogue against the all too prevalent endamebic infections in the South, I ${ }^{1}$ ventured to make trial of the old-time ipecac treatment. The powdered root, made into pill form, was employed for the purpose, each pill being provided with an enteric coating of salol, in accordance with a previous suggestion of Rogers. The striking results which attended the trial of this treatment in a series of about fifty cases were subsequently summarized in a report read before this Association in 1909. A short time previous, George Dock ${ }^{2}$ was able to report equally favorable results from observations made inde-

* Read before the Section on Gastro-Enterology and Proctology at the Sixty-Ninth Annual Session of the American Medical Association, Chicago, June, 1918

1. Simon, S. K.: Amebic Dysentery, The Journal A. M. A., N.) 6,1909 , p. 1526 .

2. Dock, G.: Tr. Am. Soc. Trop. Med., 1909. pendently in the same field. Though confirmation of these experiences was soon recorded from numerous sources, objection to the method was raised on many sides mainly on the grounds of the clinical difficulties involved in the administration of large numbers of pills at one time, many of which were found not infrequently to pass through the bowel without dissolving. Likewise, the failure to control nausea and vomiting in some patients, in spite of a liberal enteric coating of the pill, called for additional unfavorable comment. These difficulties, which even today are still not entirely under satisfactory control, served at the time to disqualify the procedure in the eyes of a considerable element of the profession. Demand was made for a less cumbersome manner of employing the curative features of ipecac without entailing such great discomfort to the patient. The answer to this demand was not long withheld. In 1911, Vedder ${ }^{3}$ and subsequently Rogers ${ }^{4}$ were both able to report success in the therapy of endamebic infections with the use of emetin, an alkaloid derived directly from the crude ipecac root. This substance, in the form of the hydrochlorid salt, they found to be especially adapted to hypodermic administration and only rarely was emesis found to occur with its use. Likewise its prompt action on the clinical disturbances of the disease was most striking, and within a very short time the virtues of the parent drug were all but completely overshadowed and forgotten. With the course of time, however, evidence has accumulated which has tended to throw more and more doubt on the justice of many of the early claims regarding the superiority of emetins over the entire ipecac root, especially in the control of the more chronic and intractable endamebic infections. While no question can be raised of the prompt amebicidal action of emetin on the free living or vegetative organisms, more extended opportunity for observation has made it increasingly clear that, in the matter of the destruction of the endamebic cysts, the alkaloidal therapy in many instances meets with marked failures, thereby opening the way for repeated recurrences of the infection. A recasting of the earlier and more optimistic views, especially in regard to the absolute and complete specificity of emetin in endamebic disease would therefore seem to be necessary.

\section{INADEQUACY OF EMETIN}

Vedder, ${ }^{5}$ himself, as early as 1913 , was willing to admit that "a large percentage of cases treated with emetin continued to harbor the Endemeba hystolytica in the encysted and most dangerous form in the feces for some time." Likewise Allan, ${ }^{6}$ Baermann, ${ }^{7}$ Gaide and Mouzels, ${ }^{8}$ and Marchoux, ${ }^{9}$ each have reported in turn unfavorable results from their experience with the emetin treatment alone, claiming that "the endameba were not expelled from the intestinal tract to the degree that the relief of clinical symptoms would lead one to expect." Still more recently, Phillips ${ }^{10}$ concluded an excellent review of the subject with the statement that "the effects of emetin are not perma-

3. Bull. Manila Med. Soc., 1911, 3, 48.

4. Therap. Gaz. 1912, 36, 837 .

5. Vedder, E. B.: Origin and Present Status of the Emetin Treatment of Amebic Dysentery, The Journal A. M. A., Feb. 14, 1914, p. 501 .

6. Allan, W.: The Emetin Treatment in Amebic Dysentery, The Journal A. M. A., March 1, 1913, p. 664.

7. Baermann: München med. Wchnschr., 1913, 60, 1132

8. Gaide and Mouzels: Bull. Soc. path. exot., 1913, 6, 491.

9. Marchoux: Bull. Soc. path. exot., 1913, 6, 313.

10. Phillips, L.: Brit. Med. Jour., 1914, \&, 1061. 
nent and that though the active amebia are killed off very quickly, the drug has no effect on the cysts." Again, G. I. Jones ${ }^{11}$ says significantly, in summarizing a large number of cases placed at his disposal, that while he considers emetin a valuable agent in the treatment of intestinal amebiasis, "the use of this drug alone will not cure amebic dysentery." This author found, in fact, that relapses soon occurred and strongly urged the employment of ipecac by mouth, as a necessary step in the achievement of complete and permanent recovery. The results of these, as likewise of numerous similar observations which have been recorded in the literature with increasing frequency in recent years would seem to point unmistakably to the conclusion that, while emetin does prove of undoubted service in the destruction of the free living endamebas, it finds a greatly restricted field of usefulness in the all-important matter of the removal of the cysts from the intestinal tract.

A close analogy exists, in fact, between the Endameba histolytica and the malarial hematozoa in the matter of their behavior to specific medication. Both organisms, in common with protozoa in general, exhibit two separate and distinct phases in their life cycle, including a free living or vegetative stage of existence which permits of free locomotion enabling the cell to obtain its nutriment, and a second state in which the vital activities of the cell are found in abeyance within the enclosure of a cyst wall (the so-called gamete). It is only in the latter state that infection is conveyed from host to host, since the vegetative organism is prone to succumb or to undergo encystment when removed from its original environment. The pathologic conditions arising in the human organism are, however, the sole result of the vital manifestations of the free living protozoon. In its encysted state the organism lies dormant, incapable of tissue harm, but offering marked resistance to external influences. It is most important to understand, in this connection, that the protozoa, once having become encysted, have acquired thereby an increased resistance to their specific drug, in sharp contrast to the ease with which the organism in its vegetative state may be reached and destroyed in the same dosage. In fact, relatively small amounts of the specific drug, instead of killing the organism outright, will often induce the obverse effect of driving it into encystment. While the immediate clinical symptoms are promptly relieved by such a process, the difficulties surrounding the complete eradication of the infection are largely increased. In regard to malarial infections, the fact has already been well established, that relatively small amounts of quinin tend to encourage the formation of gametes. Similarly, in endamebic disease, unless appreciably large doses of ipecac or of its alkaloids are employed, the free living organism seeks protection against the toxic action of the drug by enclosing itself within a cyst wall.

In the dosage found necessary for the complete overwhelming of the offending organisms, consideration must be given to the matter of the toxicity of the drug itself on the human host. While the crude ipecac, in amounts as high as 75 grains in daily doses repeated over a period of ten days, has been found singularly devoid of toxic effects, experience has shown that the alkaloids, in an equivalent dosage, possess no such immunity.

11. Jones, G. I.: The Treatment of Intestinal Amebiasis, Thz Journal A. M. A., March 20, 1915, p. 982.
RESULTS OF INVESTIGATIONS

As far back as 1817, investigations into the pharmacology of ipecac had been made by Pelletier, ${ }^{12}$ a French chemist. Pelletier believed that the root contained but one alkaloid, to which the name emetin was applied as a fit designation for the emetic properties exhibited by the drug. It was not until 1894 that Paul and Crownley ${ }^{13}$ were able to show that the root contained a second active alkaloid, to which the term cephaelin was given. Subsequently, still another alkaloid, psychotrin, has been identified and it is not improbable that the future may reveal the presence of yet another derivative. In 1895 , W ild $^{14}$ made an exhaustive study of the poisonous effect of both emetin and cephaelin on the human organism, as well as on some of the lower animals. Numerous other observations have been contributed within more recent years, serving further to enlarge our knowledge of this important subject. Perhaps the most notable among these have been the series of extensive experiments conducted by Pellini and Wallace ${ }^{15}$ in 1916 into the various toxic effects produced by emetin on the various organs of the body. As a result of their findings, they were able to formulate the following conclusions:

1. Emetin depresses, and may eventually paralyze the heart.

2. It is a powerful gastro-intestinal irritant, whether given by mouth or by subcutaneous injection.

3. It causes a definite derangement of metabolism characterized by an increase in nitrogen loss and an acidosis.

4. While in normal individuals, given moderate doses, these actions may not be of importance, in pathologic states of the circulation, intestinal tract, or metabolism, there may be a very definite source of danger.

While it is true markedly poisonous effects are rarely observed in connection with the comparatively small doses of emetin ordinarily employed in clinical practice, milder types of toxemia are by no means uncommonly encountered. Lyons, ${ }^{\mathbf{1 6}}$ for example, as early as 1915, pointed out the not infrequent occurrence of a peripheral neuritis in the course of the ordinary subcutaneous administration of emetin. In my opinion, this complication may take place even with very small doses of the drug and, while rarely of a serious character, may nevertheless give rise to a considerable degree of pain and muscular discomfort. Other sequelae resulting from the use of emetin in average doses from time to time have been recorded in the literature, such as an intractable form of watery diarrhea, gastric hyperemia and irritability, cardiac arrhythmia, including in addition the various types of skin reactions which frequently appear at the site of the injection of the drug.

The investigations made in regard to the toxicology of cephaelin hydrochlorid, while not carried out on as extensive a scale as has been the case with emetin, have nevertheless revealed a close similarity in the physiologic action of the two alkaloids. Cephaelin has been shown to possess greater emetic properties and is far more irritating to the skin when given hypodermically than is emetin. Its destructive effects on the vegetative endameba is probably equally as great as

12. Pelletier: Ann. Chem. and Phys., 1817, 4, 172.

13. Paul and Crownley: Phar. Jour., 1895, 25, 111.

14. Wild: Lancet, $1895, \mathbf{2}, 1274$.

15. Pellini and Wallace: Amer. Jour. Med. Sc., 1916, 152, 325

16. Lyons, R.: Amer. Jour. Med. Sc., 1915, 150, 97. 
that of emetin. This was strongly indicated in the ccurse of some clinical experiments I was enabled to make three years ago $^{17}$ on a limited number of adult subjects suffering with active intestinal amebiasis. However, the same failure in the matter of the destruction of the cysts was noted, as had been my experience previously with the emetin therapy.

Because of the failure of the ipecac alkaloids to remove encysted ameba from the tissues, and likewise in consideration of the toxic properties which have been shown to be inherent in these agents, attempts have been made recently to devise higher chemical formulas of the alkaloids which, it was thought, might overcome the various objectionable features of the simpler products.

Of these compounds perhaps the two most widely employed have been emetin mercuric iodid and emetin bismuthous iodid, prepared in accordance with definite formulas suggested by $\mathrm{DuMez}^{18}$ in 1915. These substances were designed for oral administration and, because of their relative insolubility, it was hoped that their emetic effect might be reduced to a minimum. However, according to most observers, this hope has scarcely been realized. Much favorable comment has nevertheless appeared in the recent literature, emanating principally from British sources, extolling the special virtue of these preparations in the more intractable forms of endamebic dysentery and claiming a particular influence in the extermination of the carriers.

In this country, Walters and $\mathrm{Koch}^{19}$ have recently published the results of experimental work carried on by them with synthetic derivatives of cephaelin, citing with particular favor cephaelin iso-amyl ether hydroiodid. They found this substance uniformly effective in destroying both the vegetative and the encystic endameba in the intestinal tract of cats. I have availed myself of the opportunity offered by Dr. Walters of trying out this preparation on human subjects, three in all, each presenting outspoken chronic endamebic infection of the bowel. Its action on the free living endameba proved it to be equal in value to that of the simple alkaloids, but again no definite effect could be noted in its action on the cysts. The dose employed was from $1 / 3$ to $2 / 3$ grain, three times a day in capsules by the mouth, and the treatment was made to cover a period of ten days in each case.

\section{IPECAC TREATMENT}

In view of the evident inadequacy of the akaloidal therapy and its failure effectually to destroy the endameba, once they have reached the encysted state, the conviction has grown increasingly strong with me that a return to the use of the original crude ipecac root should be most seriously considered by the profession at the present time. In my hands the plan first announced in 1909 of making use of ipecac in the form of enteric-coated pills has continued to yield uniformly gratifying results, both in meeting the active clinical manifestations of the disease processes and above all and perhaps of greatest importance, in the almost certain prevention of relapses which is achieved. The careful attention to detail which has been found so necessary to this plan of treatment, and which has unfortunately been an object of neglect on many sides in the past, would seem to justify a further word of

17. Simon, S. K.: Tr. Am. Soc. Trop. Med., 1916.

18. DuMez, A. G.: Philippine Med. Jour., 1915, B, 10, 73

19. Walters and Koch: Jour. Pharmacol, and Exper. Therap., 1917, $10,73$. explanation and emphasis at this time. The failure to observe the smaller details of the plan has been the cause, in fact, of much of the former prejudice surrounding the administration of the crude ipecac. First of all, it is essential that the patient be put to bed for the full course of the treatment, extending ordinarily over a period of ten days, and also that the dietary be restricted in the beginning to articles of food which leave no residue in the intestinal tract, such as broths, whey, albumin water and the various nutrient alcoholic preparations. To this list, milk is to be added only after the fifth or sixth day of treatment. A dose of castor oil should be administered on the morning of the first day of treatment and in the evening around 9 o'clock, from ten to fifteen salol-coated pills should be taken, each containing 5 grains of the powdered ipecac. The patient is advised to swallow these slowly with the help of moderate amounts of water. No nourishment is allowed for two hours preceding and likewise for six hours following the administration of the pills. Each succeeding night the same plan is to be repeated, the number of pills varying on each occasion from ten to fifteen. It may be found necessary, especially in the presence of any depressing effect, to discontinue the use of the pills for a one-night period. Each day the attending nurse keeps a record of the number of pills which might have passed undissolved in the stool, with the view of determining the total amount of ipecac retained at any stage of the treatment. A simple method of daily charting, illustrated in the accompanying table, has been devised by us for this purpose.

AMOUNT OF IPECAC ADMINISTERED AND RETAINED DAILY

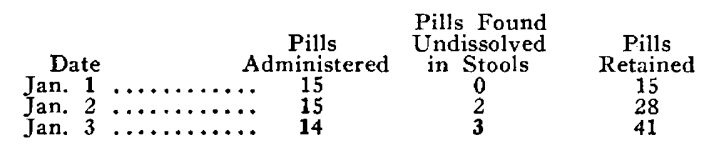

The complete dosage includes the retention of at least 100 pills, equivalent to 500 grains of the powdered ipecac. This is accomplished usually within a period of ten days and only under rare conditions must be made to extend over two weeks. Should nausea and vomiting arise as troublesome features, an extra enteric coating should be added to the pills. We have found that, under average conditions, a coating of one tenth inch of salol is ample. For reasons not altogether clear, it may happen that large numbers of the pills will pass through the intestinal tract in an undissolved state, even in spite of a diminished enteric coating. Under these circumstances, we frequently adopt the plan of making from one or two small punctures into the outer layers of the pill surface, with a small sized surgical needle. When, under rare conditions, the ipecac will not be tolerated in pill form by the patient, we may often successfully substitute the duodenal intubation method, employing for the purpose daily installations of 30 grains of the powdered ipecac suspended in water.

\section{SUPERIORITY OF IPECAC}

There remains to be considered finally the essential cause for the superior effectiveness shown by the entire ipecac root in the treatment of intestinal amebiasis, as compared with its constituent alkaloidal derivatives. From the standpoint of analogy with other therapeutic agents, it would seem that the active principles would, at least, possess a value equal to that of the parent substance. However, in the matter of the 
control of a protracted endamebic infection of the bowel, conditions of a somewhat anomalous nature are presented. The crux of the problem, to my mind, hinges on the question of the degree of concentration of the active constituents of the specific drug at the site of infection in the large bowel. When the alkaloids are administered by hypodermic injection or by mouth within the confined limits of safety, the attenuation that results in the blood stream renders the drug relatively inert when brought to bear on the infecting organisms in the intestinal wall. Under the influence of a weak dilution, the endamebas escape immediate destruction by seeking cover, so to speak, within a resistant cyst wall. Were it possible to employ massive doses of the alkaloid, prompt and rapid annihilation of the endameba might be expected to ensue. The toxic character exhibited by these substances in excessive amounts precludes, however, the employment of a large dosage. With the use of the ipecac mass orally, in daily amounts as high as 75 grains and yielding approximately 1.5 grains of total alkaloids, a concentration of the effective properties of the drug is attained in the infected colon, without any possible danger of toxemia. The patient's intestinal tract, having been rendered free by dietary restrictions, as explained in the foregoing, the pills pass quickly into the large bowel, where the salol coating is finally dissolved away in its entirety and the ipecac thus set free for absorption. It may be considered that soluble compounds of the alkaloids are formed directly within the colon, and during the process of absorption not only the vegetative endameba, but the encysted forms as well, suffer destruction in the concentrated medium. Apart from whether this explanation be considered adequate or not, it is my firm conviction, based on clinical observations extending now over several years, that the treatment of intestinal endamebiasis by means of the crude ipecac offers a most promising outlook for complete and permanent cure, not attainable with the use of the alkaloids alone, whether administered orally or hypodermically.

\section{CONCLUSIONS}

1. Both ipecac and its constituent alkaloidal derivatives, emetin and cephaelin, have been found to act specifically on the vegetative or free living forms of the Endameba histolytica.

2. The crude ipecac root in doses sufficient completely to destroy the infecting organisms is never toxic.

3. Both emetin and cephaelin frequently exhibit toxic properties in an average dosage of from 0.5 to 1 grain daily over a limited period.

4. The alkaloids alone are ineffective within safe limits of dosage in destroying the encysted forms of the Endameba histolytica.

5. The entire ipecac root, when employed under proper conditions, not only destroys the vegetative endameba but the encysted forms as well, and thereby prevents recurrences or relapses of the infection.

\section{ABSTRACT OF DISCUSSION}

Dr. Frank Smithies, Chicago: I agree with Dr. Simon that the use of crude ipecac has been effectual in the treatment of acute or chronic enterocolonic amebiasis. Perhaps Dr. Simon unintentionally neglected to state that there are instances where emetin is also of service. I came to the conclusion some years ago that large doses of ipecac administered after the method described by Dr. Simon and first suggested by Dock, aided in quickly ridding the terminal ileum and the colon of organisms, if emetin be given hypodermically at the same time. Dr. Simon explained the reason for large doses of ipecac, namely, to obtain a continued saturation of the bowel contents with the drug. Often encysted or active amebas are deep within the mucous membrane of the intestinal wall. A medicine solely in the bowel cannot reach them and consequently does not kill them. It is in this class of case that it is necessary to carry the amebacide to the organism by means of the lymph or the blood stream; therefore, it is necessary to combine the ipecac treatment with emetin given hypodermically. Unless this is done, the bowel contents will be rid of protozoa but the organisms already in the wall of the intestine will carry on their destructive work. If one uses emetin alone, and neglects to use the ipecac saturation of bowel contents, it is self-evident that sooner or later the ulcers free from amebas will become reinfected from the bowel contents. The combined treatment is successful in curing the majority of patients, and prevents recurrences.

In respect to recurrences, I would emphasize the frequent finding of viable or encysted amebas in the gallbladder, appendix or old, partly healed intestinal ulcers. In these cases it sometimes is possible to remove the foci of infection surgically. I have seen several cases where there was complete recovery after the removal of the gallbladder. Further, it is my opinion that patients are too often pronounced cured; that the stools are not examined for a sufficiently long time after the administration of specific medication. Viable amebas and cysts are often present in the stools when the individual is symptomatically well. We have the patients return once every two or three months for reexamination of freshly passed stools. We never use castor oil as a preliminary cathartic but rather a saline. This enables the exact examination of the stool microscopically.

Recently we have been using ipecac by a new method, namely, the introduction of from 2 to 4 ounces of wine of ipecac directly into the colon by rectum. This has been effective where other methods of giving ipecac have failed. We have recently had two patients under observation in whom almost constant hemorrhage produced severe anemia. In this class of case we have employed transfusion of large quantities of whole blood beneficially. This procedure should be employed more frequently in order to increase tissue defense in severe amebic enterocolitis.

Dr. Hugo A. Freund, Detroit: My observations bear out what Dr. Simon said, that occasionally patients do not respond to ipecac. Having had a somewhat unsatisfactory experience with emetin and a very happy experience with ipecac, I thought of using the duodenal tube to give the wine of ipecac, preparing the patients much the same as Dr. Simon has suggested, except that at the end of about three days I give them a small dose of opium in some form to quiet them, so they will probably be constipated for two or three days following. I pass the duodenal tube on an empty stomach, and then inject the wine of ipecac. By the instillation of relatively large doses I get satisfactory results, beginning the first day by giving 1 ounce, the next day 2 , and so on. I have given as high as 6 ounces in one instillation. I have had very satisfactory results in the fourteen cases I treated by this method. I reexamine them every three months, looking for encysted forms; and I have not seen a single relapse.

Dr. Horace W. Soper, St. Louis: Since I adopted the use of emetin intravenously $I$ have had fewer relapses. Two cases of neuritis developed, but they were mild, and very soon subsided. The emetin is injected in 1-grain doses, until the lesions have cleared up, as demonstrated by the sigmoidoscope. The lesions are very characteristic, small, elevated, grayish patches, which, when wiped off, leave a little bloody spot. The viscid mucus in these patches shows almost a pure culture of ameba. So that in the treatment of these cases we watch the lesions, and discontinue the emetin when the case is clinically improved. I give it once a week for two months, hoping to catch the encysted forms at the time of their development. The ipecac as used by Dr. Simon presents some disadvantages, inasmuch as the pills are very 
difficult to make. Dr. Simon's pharmacist has, perhaps, solved that problem. Some years ago I was unable to get a pill that would not cause nausea or that would not dissolve in the intestinal tract.

Dr. Sidney K. Simon, New Orleans: The whole aim in the treatment of an endamebic infection lies in the removal of the cysts. The destruction of the vegetative forms alone, with the relief of clinical symptoms that follows, is in itself rarely a difficult problem. Putting the patient to bed for a period of a few days will usually suffice to relieve the immediate clinical distress even without the aid of other measures. It is only in the complete destruction of the encysted forms, however, that relapses can be prevented and a permanent cure established.

In regard to the examination of the stools, we long ago aclopted the proctoscopic method in all cases of suspected protozoon infection. In this way the smears are made directly from ulcerated or denuded spots on the rectal mucosa, without the necessity of having the stools conveyed in bulk. The severe grades of anemia represent the end result of a protracted endamebic infection. With specific medication such a complication should rarely be observed. In the last few years, I have seen very few instances of severe anemia among my endameba cases.

Dr. Freund's ipecac method $I$ intend to keep in mind. However, since the method I have described this morning has been uniformly successful as a curative measure in fully 500 cases, with very infrequent, if any, relapses, other plans do not, of course, make strong appeal. In regard to the intravenous injections of emetin hydrochlorid, mentioned by Dr. Soper, I would wish to advise caution. Such a procedure, especially in the hands of the less experienced, would be capable, I think, of much abuse.

The point Dr. Soper made concerning the availability of the salol coated ipecac pill has been a bone of contention ever since the method of treatment was inaugurated. Any competent pharmacist should be able to dispense these pills satisfactorily. However, a certain amount of technic is required which the average druggist does not seem to have acquired. The pills cannot be produced in bulk by the manufacturing pharmaceutical houses, because of the fully demonstrated lack of durability of the product. The pills must be made by the individual druggist and dispensed in quantities sufficient only for the individual case.

FOCAL INFECTIONS OF THE EYE FROM THE INTESTINAL TRACT *

\section{JAMES GARFIELD DWYER, M.A., M.D. NEW YORK}

During the last few years marked attention has been paid to the consideration of infections of various parts of the body, due to a low-grade chronic infection in other parts, especially in the teeth and the tonsils. A very considerable advance has been made in the treatment of these so-called focal infections by the treatment of the original foci. The number of such cases on record is enormous and is fast increasing, and nowadays with certain infections we look for the primary cause and eradicate it if possible. Among these focal infections are some lesions of the eye that used to be grouped under a general classification, as "rheumatism," etc. We know now that there is probably no such disease as rheumatism per se, but that the symptoms classified as rheumatism are probably manifestations of some focus of infection. The work on focal infections of the eye, due to infections of the teeth and tonsils, has been reported by many observers and need not be touched on. This paper will deal with focal "Read before the Section on Ophthalmology at the Sixty-Ninth
Annual Session of the American Medical Association, Chicago, Iume, Annual infections of the eye, due in all probability to the absorption of toxins from the intestinal tract. A few suggestions will also be made as to the method of treatment now being given a trial in order to overcome these infections, since it is impossible to treat the intestine surgically in the same way teeth and tonsils are treated.

This investigation has been going on for about two years, and any statements that are made are the outcome of what we have learned in that time, and are subject to change in the future. The whole idea of presenting the paper now is to direct attention to such infections and to possible methods of treating them.

At the beginning I was confronted with the fact that no two authorities were agreed as to what the intestinal contents in general should be ; that is, the reaction, the amount of chemical constituents, the food detritus and the bacteriologic content. This was and still is a great difficulty. Some may question my interpretation of what normal intestinal contents should be, but from an examination of hundreds of samples, I have come to look for certain main characteristics.

Practically all authorities agree that normal feces is faintly alkaline to litmus, but litmus is a rough and ready method of taking the titration. With phenolphthalein the reaction is slightly acid, but only with fresh feces, because the reaction changes very rapidly on standing. The question of reaction is a very important one and one not easy to decide, as the end-point of acidity of alkalinity varies with the indicator used, namely, litmus, methyl orange, plenolphthalein, etc.

Just as in diabetes and nephritis the modern schools of medicine are aiming to test the efficiency of the body to assimilate the three food elements, namely, fats, carbohydrates and proteins, so I examined the detritus, keeping these three fundamental bases in mind. My guiding principle was to cut out of the diet the types of food that were not being assimilated, in other words, food elements whose by-products are indol, skatol and phenol. These products are normally present and unless abnormal in amount can be disregarded, but as the former two are known to be absorbed into the system, appearing as indican in the urine, and as they are products of protein metabolism, I paid special attention to them, as will be noted later.

\section{BACTERIOLOGY}

Here I was confronted with the biggest problem of all, as the intestine normally contains many and various forms of bacteria. I made no endeavor to identify the various forms of bacteria present, as that would have been impossible; but bearing in mind the work of various investigators, especially.Metchnikoff, I paid special attention to the presence or absence of the colon bacillus. Now, the colon bacillus appears in the intestine a few days after birth, and is usually found there throughout life. Again, the colon bacillus is gram negative, that is, it stains a different color from that of the other bacteria usually found, the latter being gram positive with very few exceptions. Hence we have a ready means of comparison. The ptomain producers are usually gram-positive large bacilli, producing either acid or alkali, according to the group. Many of them are of the anaerobic varieties, such as the Bacillus aerogenes-capsulatus.

Keeping these facts in mind and trying to narrow the whole affair down to a practical working basis, I carried out the following routine: The intestinal contents were examined as to their reaction, their content 